\title{
Outcome of Patients with Low-Flow/Low-Gradient Severe Aortic Stenosis Who Underwent Aortic Valve Replacement
}

\author{
*Xingli Fan, MD, ${ }^{*}$ Jiajun Zhang, MD,${ }^{1}$ Chong Wang, MD, PhD, ${ }^{2}$ Hongmei Chong, MA, ${ }^{3}$ \\ Guanxin Zhang, MD, PhD, ${ }^{1}$ Qing Xue, MD, PhD, ${ }^{1}$ Yangfeng Tang, MD, $\mathrm{PhD},{ }^{1}$ Lin Han, MD, $\mathrm{PhD}^{1}$ \\ ${ }^{1}$ Department of Cardiovascular Surgery, Changhai Hospital, Second Military Medical University, Shanghai; ${ }^{2}$ Department of \\ Cardiovascular Surgery, Xiongke Hospital, Shanghai Jiao Tong University, Shanghai; ${ }^{3}$ EA-Sailing (Beijing) Culture Exchange \\ Co, Ltd, Beijing, China
}

\section{ABSTRACT}

Background: It is well-documented that stroke volume and gradient are indexed to classify patients with aortic stenosis into several phenotypes. The purpose of the present study was to estimate the impact of stroke volume and gradient on the clinical outcome of patients with AS who have undergone aortic valve replacement.

Methods: A total of 154 consecutive patients were studied. They all had severe aortic stenosis (aortic valve area [AVA] $\leq 1 \mathrm{~cm}^{2}$, left ventricular ejection fraction $[\mathrm{LVEF}] \geq 50 \%$ ) and underwent aortic valve replacement (AVR) from January 1, 2004 to December 31, 2010. Clinical and echocardiography data was collected. According to stroke volume index (SVi), low flow (LF, $\mathrm{SVi}<35 \mathrm{~mL} / \mathrm{m}^{2}$ ) and normal flow (NF, SVi $\geq 35$ $\mathrm{mL} / \mathrm{m}^{2}$ ) were defined, and according to transvalvular pressure gradient, low gradient (LG, gradient $<40 \mathrm{mmHg}$ ) and high gradient ( $\mathrm{HG}$, gradient $\geq 40 \mathrm{mmHg}$ ) were also defined. Based on the above classification, patients were separated into four groups: NF/HG (59 patients), NF/LG (30 patients), LF/HG (40 patients) and LF/LG (25 patients). To estimate the discrepancy between patients with bicuspid aortic valve (BAV) and normal 3-leaflets aortic valve, 154 cases were divided into 2 groups: BAV group and 3-leaflets group. In-hospital mortality and overall survival were followed up. The risk factors of in-hospital mortality and overall survival were estimated by logistic regression analysis and Cox regression analysis.

Results: The mean follow-up time was $59 \pm 32$ months of 154 patients among whom the in-hospital mortality of NF/HG was $1.7 \%$ compared with NF/LG (6.7\%), LF/HG $(12.5 \%)$ and LF/LG (10.5\%). The overall survival rates among the four groups were NF/HG (72\%), NF/LG (92\%), LF/HG (55\%) and LF/LG (84\%). The 5-year survival rate

Received November 3, 2016; accepted February 9, 2017.

Xingli Fan and Fiajun Zhang contributed equally to this work. This work was supported by the grants from National Natural Science Foundation of China (No. 81370471 to Lin Han; No. 81541003 to Guanxin Zhang) and Shanghai Science Committee Foundation (No. 15 ZR1413400 to Guanxin Zhang).

Correspondence: Lin Han, MD, PbD, Department of Cardiovascular Surgery, Changhai Hospital, Second Military Medical University, Changhai Road, Yangpu District, Shanghai, China;+021-31161763 (e-mail:sh_banlin@163.com). was lower in the BAV group than in the 3-leaflets group (78\% and $93 \% ; P<.05)$. The independent value for the in-hospital mortality included atrial fibrillation, concomitant coronary artery bypass graft, cardiac index, and bicuspid aortic valve. The independent factors for the overall survival included valvulo-arterial impedance, time of cardiopulmonary bypass, atrial fibrillation, bicuspid aortic valve, and concomitant coronary artery bypass graft.

Conclusion: The in-hospital outcome of LF/LG is worse than NF/HG and NF/LG, but similar to LF/HG. For the overall outcome, LF/LG is better than NF/HG and LF/HG, but worse than NF/LG. Patients with BAV exhibit worse survival compared to 3 -leaflets aortic valve.

\section{INTRODUCTION}

Aortic stenosis (AS) is a serious health problem worldwide. The most crucial pathological determinant is a chronic pressure overload onto the left ventricule caused by the calcified, immobile aortic valve, leading to concentric hypertrophy and myocardial fibrosis. Severe AS has been defined as aortic valve area $(\mathrm{AVA})<1 \mathrm{~cm}^{2}$ with a mean gradient $>40 \mathrm{mmHg}$. It is common for a patient to meet 1 or 2 of these criteria. Recently, there has been an increasing number of studies regarding low flow/low gradient (LF/LG) [Dumesnil 2010; Minners 2010; Voisine 2006]. To further define the hemodynamic features of severe AS, a recent flow-gradient classification has been proposed, incorporating both mean gradient and stroke volume. Aortic valve replacement (AVR), either surgical or by a transcatheter approach, is the sole definitive treatment for aortic stenosis that results in immediate relief of mechanical obstruction. AVR can improve outcome of patients with AS; however, there is controversy about its effect on AS patients with LF/LG despite normal LVEF (PLG-SAS) [Adda 2012; Clavel 2012; Hachicha 2007; Jander 2011].

Accordingly, the aim of the present investigation was to determine the difference in clinical characteristics of patients with severe AS and preserved EF and the effects of AVR according to stroke volume and aortic valve gradient.

\section{MATERIALS AND METHODS}

A total of 863 consecutive patients were retrospectively studied. They had severe aortic stenosis and underwent aortic 
Table 1. Basic Clinical Characteristics and Echocardiography Data

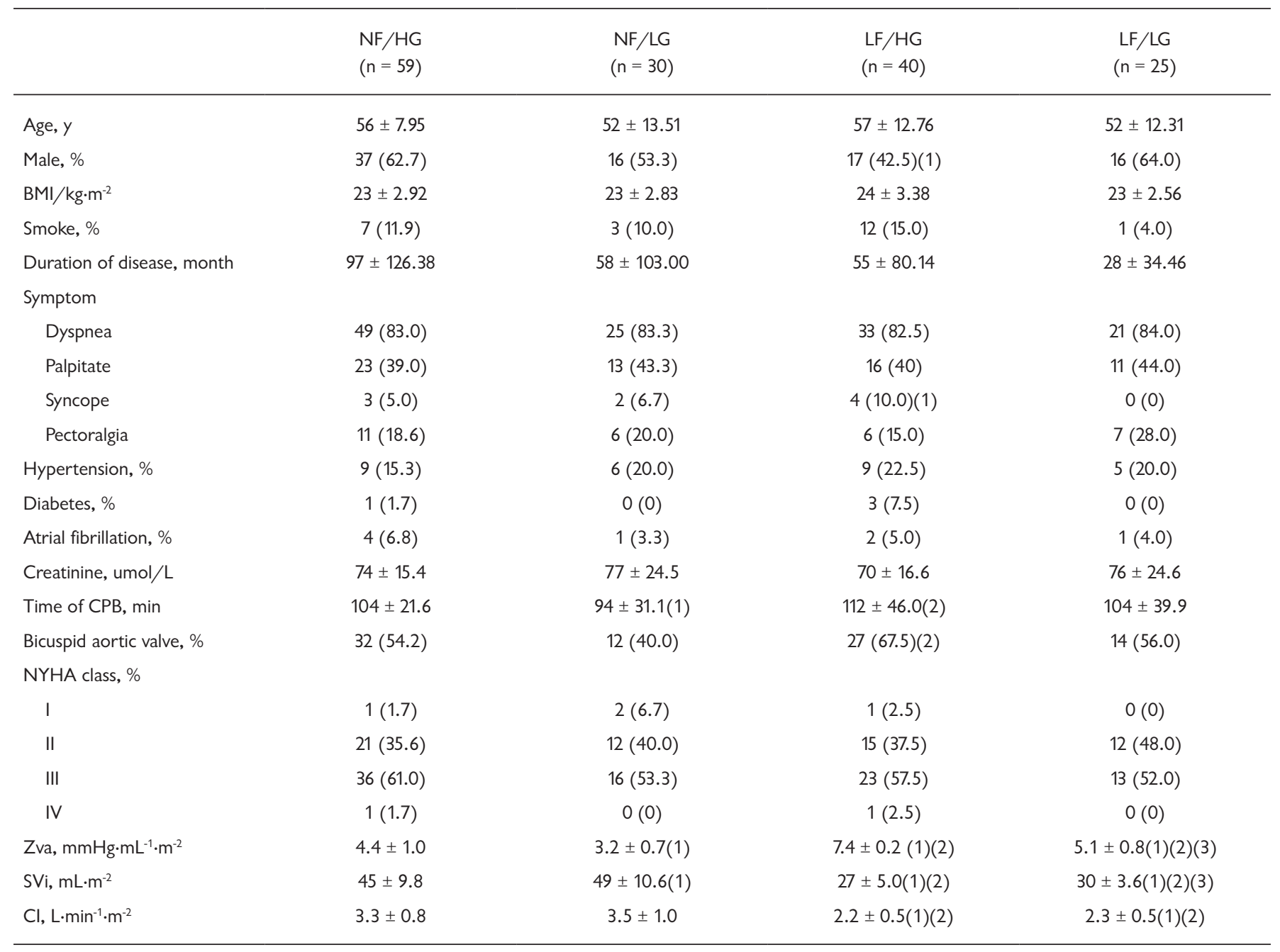

Compared to NF/HG group (1) $P<.05$ compared to NF/LG group (2) $P<.05$ compared to LF/HG group (3) $P<.05$.

valve replacement (AVR) from January 1, 2004 to December 31,2010 . Patients aged $<18$, AVA $>1 \mathrm{~cm}^{2}, \mathrm{LVEF}<50 \%$, moderate to severe regurgitation, infectious endocarditis, contemporaneous other valve disease, cardiac surgery history and valve prosthesis were excluded. After exclusion, 154 patients were eligible for study and underwent AVR. This study was approved by the Ethics Committee of Changhai Hospital, Shanghai, China.

Baseline clinical data and echocardiography data were obtained by medical records and databases. Baseline clinical data consisted of age, sex, height, weight, body surface area (BSA), basal metabolic rate (BMI), smoke, duration of disease, symptoms (dyspnea, syncope, pectoralgia), hypertension, diabetes, atrial fibrillation, lung function, creatinine, time of cardiopulmonary bypass (CPB), bicuspid aortic valve, contemporaneous CABG, and NYHA class. 154 patients $(100 \%)$ underwent echocardiography assessment preoperatively while systolic arterial pressure (SAP) and diastolic arterial pressure (DAP) were detected. Echocardiography assessment was completed by a doctor in the echocardiography department of Changhai Hospital, China. Zva was calculated by the following formula: Zva = $(\mathrm{SAP}+\mathrm{MG}) / \mathrm{SVI}$.

Statistical analysis was performed with SAS software, Version 9.1 (SAS Institute, Cary, NC, USA). All continuous variables were expressed as mean \pm standard deviation and were compared according to the Student $\mathrm{t}$ test or Wilcoxon rank-sum test between two groups. Categorical variables were expressed as percentages and compared according to Pearson chi-square test. Comparisons of continuous variables among the four groups were performed with Kruskal-Wallis test. Logistic regression analysis was used to screen the risk factors of the mortality after AVR (SLE $=0.10$, SLS $=0.15)$. The survival of patients from different groups was obtained by Kaplan-Meier estimates. Finally, the Cox proportional-hazards model was 


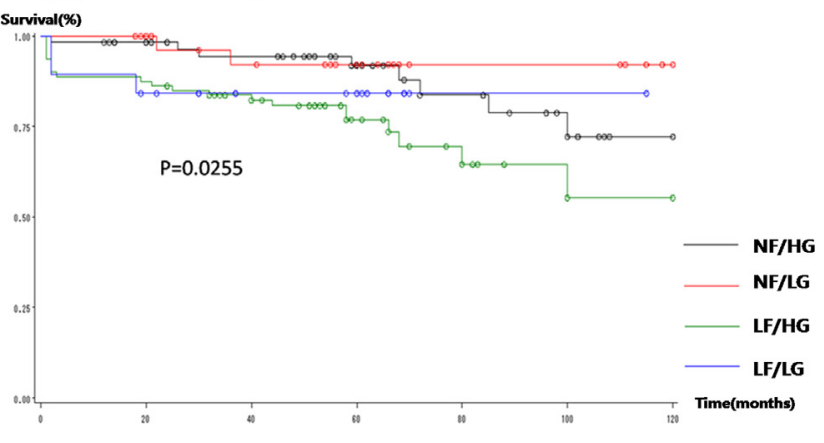

Figure 1. Overall survival to death. The LF/HG group had the worst survival $(P=.0255)$.

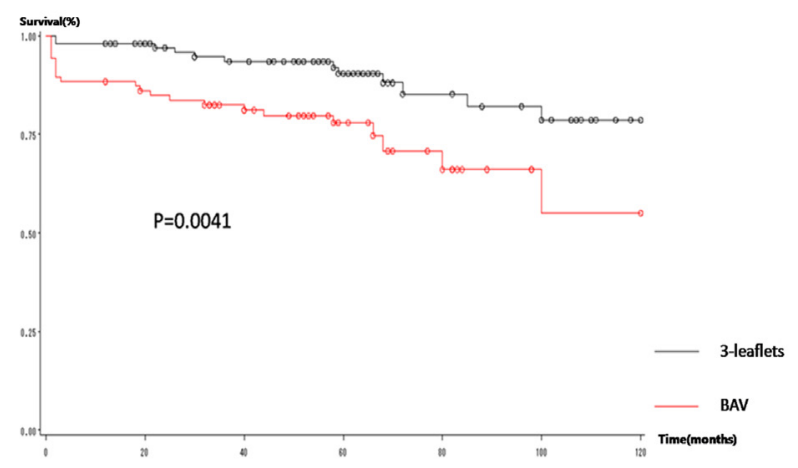

Figure 2. Overall survival to death. The BAV group had worse survival as compared to the 3 -leaflets group $(P=.0041)$.

used to assess the effect of clinical and echocardiography variables on survival. A $P$ value $\leq .05$ was considered statistically significant.

\section{RESULTS}

Patient characteristics are shown in Table 1 . The rate of female and syncope were significantly higher in the LF/HG group compared to the NF/HG group $(57.5 \%$ and $37.3 \%$, $P<.05 ; 10.0 \%$ and $5.0 \% ; P<.05)$. Among the NF/HG group, LF/HG group, and NF/LG group, time of CPB $(104 \pm 21.6$ min, $112 \pm 46.0 \mathrm{~min}$ and $94 \pm 31.1 \mathrm{~min} ; P<.05)$ was shortest in the NF/LG group. Zva was significantly higher in the LF/ LG group compared to the NF/HG group and the NF/LG group $\left(5.1 \pm 0.8 \mathrm{mmHg} \cdot \mathrm{mL}^{-1} \cdot \mathrm{m}^{-2}, 4.4 \pm 1.0 \mathrm{mmHg} \cdot \mathrm{mL}^{-1} \cdot \mathrm{m}^{-2}\right.$ and $\left.3.2 \pm 0.7 \mathrm{mmHg} \cdot \mathrm{mL}^{-1} \cdot \mathrm{m}^{-2} ; P<.05\right)$. Zva remained highest in the LF/HG group $\left(7.4 \pm 0.2 \mathrm{mmHg} \cdot \mathrm{mL}^{-1} \cdot \mathrm{m}^{-2} ; P<.05\right)$ and lowest in the NF/LG group $(P<.05)$. In the 4 groups, Svi was highest in the NF/LG group $\left(49 \pm 10.6 \mathrm{~mL} \cdot \mathrm{m}^{-2} ; P<.05\right)$, and lowest in the LF/HG group $\left(27 \pm 5.0 \mathrm{~mL} \cdot \mathrm{m}^{-2} ; P<.05\right)$. SVi was significantly lower in the LF/LG group than in the NF/ HG group $\left(30 \pm 3.6 \mathrm{~mL} \cdot \mathrm{m}^{-2}\right.$ and $\left.45 \pm 9.8 \mathrm{~mL} \cdot \mathrm{m}^{-2} ; P<.05\right)$. CI was significantly lower in the LF/HG group and LF/LG
Table 2. Risk Factors of In-Hospital Mortality

\begin{tabular}{lccc}
\hline Model & HR & $95 \% \mathrm{Cl}$ & $P$ \\
\hline AF & 17.525 & $2.469-24.388$ & .0042 \\
CO-CABG & 6.092 & $1.339-27.724$ & .0194 \\
SVi & 0.376 & $0.174-0.813$ & .0129 \\
Creatinine & 8.279 & $0.994-68.94$ & .0506 \\
BAV & 5.927 & $0.943-37.274$ & .0578 \\
\hline
\end{tabular}

$\mathrm{AF}$ indicates atrial fibrillation; $\mathrm{CO}-\mathrm{CABG}$, concomitant coronary artery bypass graft; SVi, stroke volume index; BAV, bicuspid aortic valve; HR, hazard ratio; $\mathrm{Cl}$, confidence interval.

group $\left(3.3 \pm 0.8 \mathrm{~L} \cdot \mathrm{min}^{-1} \cdot \mathrm{m}^{-2}, 3.5 \pm 1.0 \mathrm{~L} \cdot \mathrm{min}^{-1} \cdot \mathrm{m}^{-2}\right.$ and $2.2 \pm$ $\left.0.5 \mathrm{~L} \cdot \mathrm{min}^{-1} \cdot \mathrm{m}^{-2}, 2.3 \pm 0.5 \mathrm{~L} \cdot \mathrm{min}^{-1} \cdot \mathrm{m}^{-2} ; P<.05\right)$ compared to the NF/HG group and NF/LG group.

For the 154 patients, the mean follow-up time was $59 \pm 32$ months. In the in-hospital mortality, NF/HG was $1.7 \%$ compared with NF/LG (0), LF/HG (15.0\%), and LF/LG (12.0\%). The overall survival rates for the 4 groups were NF/HG (72\%), NF/LG (92\%), LF/HG (55\%), and LF/LG (84\%) (Figure 1). The BAV group was characterized by high overall mortality (Figure 2). The 5 -year survival rate was lower in the BAV group than in the 3 -leaflets group $(78 \%$ and $93 \% ; P<.05)$. The risk factors of in-hospital mortality and overall mortality are shown in Table 2 and Table 3. In the study, atrial fibrillation, concomitant coronary artery bypass graft, cardiac index, and bicuspid aortic valve were the independent values for the in-hospital mortality; and cardiac index, valvulo-arterial impedance, time of cardiopulmonary bypass, atrial fibrillation, bicuspid aortic valve and concomitant coronary artery bypass graft were the independent factors for the overall survival.

\section{DISCUSSION}

Low flow/low gradient and normal LV function are found in $5-25 \%$ among patients with AS. There is controversy for outcome of patients with PLG-SAS. Lancellotti et al used B-type natriuretic peptide (BNP) to estimate the outcome of LF/LG AS; the results indicated that the LF/LG and LF/HG groups had higher BNP and lower cardiac event-free survival compared with NF/HG and NF/LG groups [Lancellotti 2012]. Maes et al indicated that the paradoxical LG severe AS was a less malignant form of AS compared with HG severe AS, and its spontaneous outcome was better [Maes 2014]. Interestingly, Jander et al concluded that patients with LG AS and normal LVEF had an outcome similar to the patients with moderate stenosis $\left(\mathrm{AVA}=1.0-1.5 \mathrm{~cm}^{2} ; \mathrm{MG}=25-40 \mathrm{mmHg}\right.$ ) [Jander 2011].

$\mathrm{LV}$ function is an important factor that influences outcome of patients with AS who undergo AVR. The incidence of syncope is high in the LF/LG group, which may be due to concentric hypertrophy of $\mathrm{LV}$, smaller volume and low SV 
Table 3. Risk Factors of Overall Mortality

\begin{tabular}{lccc}
\hline Model & $\mathrm{HR}$ & $95 \% \mathrm{Cl}$ & $P$ \\
\hline $\mathrm{SVi}$ & 0.35 & $0.166-0.738$ & .0059 \\
$\mathrm{Zva}$ & 1.122 & $0.969-1.299$ & .1247 \\
Duration of CPB & 1.007 & $1.001-1.013$ & .021 \\
Hypertension & 0.533 & $0.228-1.25$ & .148 \\
$\mathrm{AF}$ & 3.697 & $1.029-13.285$ & .0451 \\
$\mathrm{BAV}$ & 2.612 & $1.144-5.965$ & .0227 \\
CO-CABG & 11.523 & $4.979-26.668$ & $<.0001$ \\
\hline
\end{tabular}

SVi indicates stroke volume index; Zva, valvulo-arterial impedance; CPB, cardiopulmonary bypass; $\mathrm{AF}$, atrial fibrillation; BAV, bicuspid aortic valve; CO-CABG, concomitant coronary artery bypass graft; $\mathrm{HR}$, hazard ratio; $\mathrm{Cl}$, confidence interval.

[Clavel 2015]. More calcific leaflets, and restricted leaflets activity may have contributed to worse prognosis in the LF/ LG group than in the NF/HG group [Nishimura 2014]. In this study, patients with LF/LG had small AVA and small LV cavities. This indicates that these entities may have concentric remodeling of $\mathrm{LV}$, reduced cavity size, and impaired diastolic and systolic function, which were unrevealed by LVEF. The condition suggests that the LVEF could not reveal the real LV systolic function. High valvulo-arterial impedance is also present in patients with LF/LG. It is reasonable that the high LV afterload leads to the adjustment of LV geometry, eg reduced cavity, changes in myocardial thickness, and concentric remodeling that will influence incidence of cardiac events. Recent studies have found the incidence of $\mathrm{LV}$ dysfunction increased 4-fold when $Z_{v a}>5 \mathrm{mmHg} /\left(\mathrm{mL} \cdot \mathrm{m}^{2}\right)$ [Eleid 2013; Hachicha 2009]. Coincidently, Zva is an independent risk factor of survival after AVR.

In the present study, bicuspid aortic valve (BAV) has a high prevalence of $55.2 \%$ in all 154 patients. This ascertainment is inconsistent with recent studies. BAVs usually exhibit normal function at birth and during early life, but they can be associated with significant aortic valve disease prior to adulthood [Mathieu 2015]. Later in life BAVs are associated with substantial morbidity [Michelena 2008]. In particular, BAVs are predisposed to progressive calcification, grossly identical to that occurring in tricuspid aortic valves. Calcific stenosis of a BAV is generally accelerated approximately a decade earlier than with surgery. Calcified BAVs often become clinically important in patients as young as 50 years old; hence, it is reasonable that cases in this study presented younger. Structural abnormalities of the aortic wall commonly accompany BAV, and this may potentiate both aortic dilatation and aortic dissection [Mathieu 2015]. In the present study, patients with BAV are associated with lower survival rates. It may be due to the high prevalence of reoperation of dilated ascending aorta after isolated AVR.

This study demonstrates that patients with $\mathrm{LF} / \mathrm{HG}$ are predisposed to the highest mortality among 4 groups, and higher prevalence of BAV in patients with $\mathrm{LF} / \mathrm{HG}$ compared with NF/LG. Meanwhile, as logistic regression analysis is used to estimate the predictors of mortality, BAV is an independent predictor of in-hospital and long-term death. The AVA in patients with LF/HG was remarkably smaller than other classification patterns, which suggests that the impact of BAV on aortic valve and gradient may be worse. The smaller AVA and higher gradient contribute to more myocardial thickening, greater concentric remodeling, and higher Zva. This explains the worse outcome of patients with LF/HG, LF/LG.

This study has a number of limitations. As this is a retrospective study, the data of patients who did not receive AVR are unavailable, and this might impact the outcome evaluation of patients after AVR. The sample size of the study is small which may lead to bias.

\section{Conclusion}

The in-hospital outcome of LF/LG was worse than NF/ HG and NF/LG, but similar to LF/HG. For the overall outcome, LF/LG was better than NF/HG and LF/HG, but worse than NF/LG. Patients with BAV exhibit worse survival compared to with 3-leaflets aortic valve.

\section{Acknowledgement}

The authors would like to thank Er song Wang, MD and $\mathrm{Yi} \mathrm{Qu}, \mathrm{MD}$ for their assistance in the echocardiography data collection; and Chong Wang, MD for supporting data collection.

\section{REFERENCES}

Adda J, Mielot C, Giorgi R, et al. 2012. Low-flow, low-gradient severe aortic stenosis despite normal ejection fraction is associated with severe left ventricular dysfunction as assessed by speckle-tracking echocardiography: a multicenter study. Circulation Cardiovasc Imag 5:27-35.

Clavel MA, Dumesnil JG, Capoulade R, Mathieu P, Senechal M, Pibarot P. 2012. Outcome of patients with aortic stenosis, small valve area, and low-flow, low-gradient despite preserved left ventricular ejection fraction. J Am Coll Cardiol 60:1259-67.

Clavel MA, Berthelot-Richer M, Le Ven F, et al. 2015. Impact of classic and paradoxical low flow on survival after aortic valve replacement for severe aortic stenosis. J Am Coll Cardiol 65:645-53.

Dumesnil JG, Pibarot P, Carabello B. 2010. Paradoxical low flow and/ or low gradient severe aortic stenosis despite preserved left ventricular ejection fraction: implications for diagnosis and treatment. Eur Heart J 31:281-9.

Eleid MF, Sorajja P, Michelena HI, Malouf JF, Scott CG, Pellikka PA. 2013. Flow-gradient patterns in severe aortic stenosis with preserved ejection fraction: clinical characteristics and predictors of survival. Circulation 128:1781-9.

Hachicha Z, Dumesnil JG, Bogaty P, Pibarot P. 2007. Paradoxical lowflow, low-gradient severe aortic stenosis despite preserved ejection fraction is associated with higher afterload and reduced survival. Circulation 115:2856-64.

Hachicha Z, Dumesnil JG, Pibarot P. 2009. Usefulness of the valvuloarterial impedance to predict adverse outcome in asymptomatic aortic stenosis. J Am Coll Cardiol 54:1003-11. 
Jander N, Minners J, Holme I, et al. 2011. Outcome of patients with lowgradient "severe" aortic stenosis and preserved ejection fraction. Circulation 123:887-95.

Lancellotti P, Magne J, Donal E, et al. 2012. Clinical outcome in asymptomatic severe aortic stenosis: insights from the new proposed aortic stenosis grading classification. J Am Coll Cardiol 59:235-43.

Maes F, Boulif J, Pierard S, et al. 2014. Natural history of paradoxical lowgradient severe aortic stenosis. Circulation Cardiovasc imag 7:714-22.

Mathieu P, Bosse Y, Huggins GS, et al. 2015. The pathology and pathobiology of bicuspid aortic valve: State of the art and novel research perspectives. J Path Clin Research 1:195-206.

Michelena HI, Desjardins VA, Avierinos JF, et al. 2008. Natural history of asymptomatic patients with normally functioning or minimally dysfunctional bicuspid aortic valve in the community. Circulation 117:2776-84.

Minners J, Allgeier M, Gohlke-Baerwolf C, Kienzle RP, Neumann FJ, Jander N. 2010. Inconsistent grading of aortic valve stenosis by current guidelines: haemodynamic studies in patients with apparently normal left ventricular function. Heart 96:1463-8.

Nishimura RA, Otto CM, Bonow RO, et al. 2014. 2014 AHA/ACC guideline for the management of patients with valvular heart disease: a report of the American College of Cardiology/American Heart Association Task Force on Practice Guidelines. J Thorac Cardiovasc Surg 148:e1-e132.

Voisine P, Mathieu P, Doyle D, et al. 2006. Influence of time elapsed between myocardial infarction and coronary artery bypass grafting surgery on operative mortality. Eur J Cardiothorac Surg 29:319-23. 